\title{
Severely calcified coronary stenoses: novel challenges, old remedy
}

\author{
Giuseppe Di Gioia ${ }^{1,2}$, Carmine Morisco², Emanuele Barbato ${ }^{1,2}$ \\ ${ }^{1}$ Cardiovascular Research Center Aalst, Belgium \\ ${ }^{2}$ Department of Advanced Biomedical Sciences, University of Naples, Federico II, Naples, Italy
}

Adv Interv Cardiol 2018; 14, 2 (52): 115-116 DOI: https://doi.org/10.5114/aic.2018.76400

The presence of severe calcifications in coronary artery stenoses makes percutaneous revascularizations particularly challenging, and represents the most frequent cause of stent failure. Inability to implant or properly expand the stent is not infrequent with heavily calcified coronary stenoses, leading to increased stent restenosis or thrombosis. For these reasons, surgical revascularization has been traditionally preferred in patients with heavily calcified coronary lesions. Due to the ageing of the patients undergoing coronary angiography, percutaneous coronary intervention $(\mathrm{PCI})$ is increasingly attempted in these patients following the improved performance of novel drug-eluting stents. Yet, a very old therapy is being used to address this novel challenge, namely rotational atherectomy (RA). Rotational atherectomy was introduced in interventional cardiology in 1988, even before the widespread availability of bare metal stents. Thirty years after its introduction, this technology has basically remained the same, even if the technique has evolved. The old concept of debulking has given way to the modern plaque modification technique, which means in practice applying the least ablation force needed in order to ease stent deployment and implantation [1]. Current RA technique has improved $\mathrm{PCl}$ outcomes, as suggested by several studies [2-4]. Nevertheless, RA has remained largely underused, representing 1 to $5 \%$ of the total PCl volume [1]. Possible reasons for this limited adoption include underestimation of calcification in coronary angiography, perceived complexity of the technique and potential RA-related complications, which are mentioned in the two recent observational analyses published in this issue of the journal $[5,6]$.

In the first series of 156 consecutive patients treated with RA at a high volume $\mathrm{PCl}$ center, no difference was found in terms of in-hospital complications or 12-month clinical outcome between elective and bail-out RA [5]. Of note, only $27.6 \%$ of the patients received elective RA, while in the vast majority RA was carried out after unsuccessful traditional $\mathrm{PCI}$. On one hand, this can probably be explained by the reluctance of the operators to readily perform a technique that might be perceived as complex and potentially cumbersome, as suggested by the fact that even in the group of elective RA, the latter was performed at the occasion of a staged procedure in most of the cases. On the other hand, the higher rate of bailout RA underscores how challenging it might be to clearly anticipate the severity and extent of coronary calcification in the angiogram. The operators of this registry should be commended for their foresight to have stopped balloon PCI before causing major vascular complications (e.g. dissections or rupture) that might have significantly hampered coronary flow and the attempt to stage the RA procedure.

In the ORPKI Polish National dataset [6], 245 patients with ST-elevated myocardial infarction (STEMI) or non-ST-elevated myocardial infarction (NSTEMI) treated with RA between 2015 and 2016 were compared with 91,985 contemporary patients treated without RA. As expected, patients treated with RA had a higher rate of complex coronary disease such as left main stenosis or multivessel disease. This study showed that RA-aided $\mathrm{PCl}$ is technically feasible in selected patients with acute myocardial infarction and does not negatively affect angiographic success and periprocedural complications. These data therefore support the judicious use of RA even in thrombotic lesions, which were perceived in the past as an absolute contraindication to the technique. This finding is in line with the previous ROTATE ACS registry that retrospectively compared 484 patients with non-ST-elevated acute coronary syndrome (NSTE-ACS)

\section{Corresponding author:}

Giuseppe Di Gioia, Department of Advanced Biomedical Sciences, University of Naples, Naples, Italy, e-mail: di-gioia@libero.it Received: 12.06 .2018 , accepted: 12.06 .2018$. 
with 824 patients with stable angina [7]. Even here, no differences were found in post-procedural TIMI flow and angiographic success, although more procedural complications were reported in the NSTE-ACS group. Despite these encouraging results, caution should always be exercised in case of thrombotic lesions, trying to restore antegrade flow at the time of the acute procedure, while attempting to stage RA a few days after the acute event in case of suboptimal balloon lesion preparation.

\section{Conflict of interest}

The authors declare no conflict of interest.

\section{References}

1. Barbato E, Carrié D, Dardas P, et al. European Association of Percutaneous Cardiovascular Interventions. European expert consensus on rotational atherectomy. Eurolntervention 2015; 11: 30-6.

2. Kawamoto H, Latib A, Ruparella N, et al. In-hospital and midterm clinical outcomes of rotational atherectomy followed by stent implantation: the ROTATE multicentre registry. Eurolntervention 2016; 12: 1448-56

3. Abdel-Wahab M, Richardt G, Joachim Büttner H, et al. High-speed rotational atherectomy before paclitaxel-eluting stent implantation in complex calcified coronary lesions: the randomized ROTAXUS (Rotational Atherectomy Prior to Taxus Stent Treatment for Complex Native Coronary Artery Disease) trial. JACC Cardiovasc Interv 2013; 6: 10-9.

4. De Waha S, Allali A, Büttner HJ, et al. Rotational atherectomy before paclitaxel-eluting stent implantation in complex calcified coronary lesions: two-year clinical outcome of the randomized ROTAXUS trial. Catheter Cardiovasc Interv 2016; 87: 691-700.

5. Gorol J, Tajstra M, Hudzik B, et al. Comparison of outcomes in patients undergoing rotational atherectomy after unsuccessful coronary angioplasty versus elective rotational atherectomy. Adv Interv Cardiol 2018; 14: 128-34.

6. Januszek R, Siudak Z, Dziewierz A, et al. Bailout rotational atherectomy in patients with myocardial infarction is not associated with increased periprocedural complication rate or poorer angiographic outcomes in comparison to elective procedures (from the ORPKI Polish National Registry 2015-2016). Adv Interv Cardiol 2018; 14: 135-43.

7. Iannaccone M, Piazza F, Boccuzzi GG, et al. ROTational AThErectomy in acute coronary syndrome: early and midterm outcomes from a multicentre registry. Eurolntervention 2016; 12: 1457-64. 